\title{
Hallazgos radiológicos en sistema nervioso central (SNC) del síndrome de MELAS: reporte de caso
}

Radiological findings of MELAS syndrome in central nervous system (CNS): case report

Oscar Mauricio Romero Osorio (1), Ana María Ayala Copete (2).

\begin{abstract}
RESUMEN
Presentamos el caso clínico de una paciente adulta joven con episodios recurrentes sugestivos de ataque cerebrovascular, con cambios radiológicos típicos de enfermedad de MELAS con confirmación genética de mutación en el gen A3243G.
\end{abstract}

PALABRAS CLAVE: MELAS, resonancia magnética, espectroscopia, TAC, RM funcional (DeCS).

\section{SUMMARY}

A clinical case of a young adult patient with recurrent episodes suggestive of stroke, with typical radiological changes of MELAS disease with genetic confirmation of mutation in A3243G gene.

KEY WORDS: MELAS, Magentic resonance, Spectroscopy, CT Scan, Functional MRI (MeSH).

\section{Presentación del caso}

Paciente de 53 años, odontóloga con el siguiente cuadro de inicio al despertar: desorientación, irritabilidad, conductas inapropiadas y lenguaje incoherente. Un familiar notó la dificultad para encontrar objetos, tropezar al caminar, dificultad para ejecutar labores, requiriendo incluso asistencia para vestir.

Como antecedente, presentó dos eventos interpretados como ACV a los 40 años, cuya manifestación fue recorte campimétrico izquierdo, pérdida de visión de colores, dificultad en ejecución y secuenciación de acciones, asociado a debilidad del lado izquierdo. En ese entonces se inició manejo anti-isquémico y rehabilitación persistiendo con secuelas cognitivas. No hay historia de debilidad, otros síntomas sistémicos, ni antecedentes familiares relevantes.

Al examen neurológico presentó: hipoprosexia, lenguaje con bloqueos, comprende órdenes, nomina y repite con dificultad, apraxia ideomotora, discalculia, agnosia digital, agnosia para colores y anosognosia, extinción al doble estimulo táctil. Presentó conductas de utilización, prensión palmar bilateral. En examen de pares craneales: hemianopsia derecha e hipoacusia neurosensorial bilateral. No hay alteración motora, en coordinación ni otras alteraciones en examen físico general o neurológico.

Bajo sospecha de ACV, se inició estudio con TAC de cráneo simpe (figura 1) encontrando hipodensidad cortico-subcortical temporal, parietal y occipital izquierda, calcificaciones gangliobasales y pérdida de volumen en región parieto - occipital derecha. Los estudios de factores de riesgo vascular fueron negativos para fuentes de embolismo y, se realizó RM cerebro (figura 2), encontrando las secuencias T2 y FLAIR (Fluid Attenuation Inversión Recovery) una extensa lesión hiperintensa de predominio cortical en la región parieto - temporo - occipital izquierda sin respetar territorio vascular. En las secuencias de difusión (DWI y mapa de ADC) presenta restricción de señal. Se observó adicionalmente incremento del tamaño del asta posterior del ventrículo lateral derecho con atrofia de la corteza adyacente, sin evidencia de una franca lesión malácica. La secuencia de susceptibilidad magnética (SWI) muestra las calcificaciones

(1) Médico Pontificia Universidad Javeriana. Residente de neurología Pontificia Universidad Javeriana. Bogotá, Colombia.

(2) Estudiante de medicina, Facultad de Medicina, Pontificia Universidad Javeriana. Bogotá, Colombia

Recibido: 1/11/16. Aceptado: 3/02/17.

Correspondencia: Oscar Mauricio Romero Osorio, oscar_ro14@hotmail.com

22 


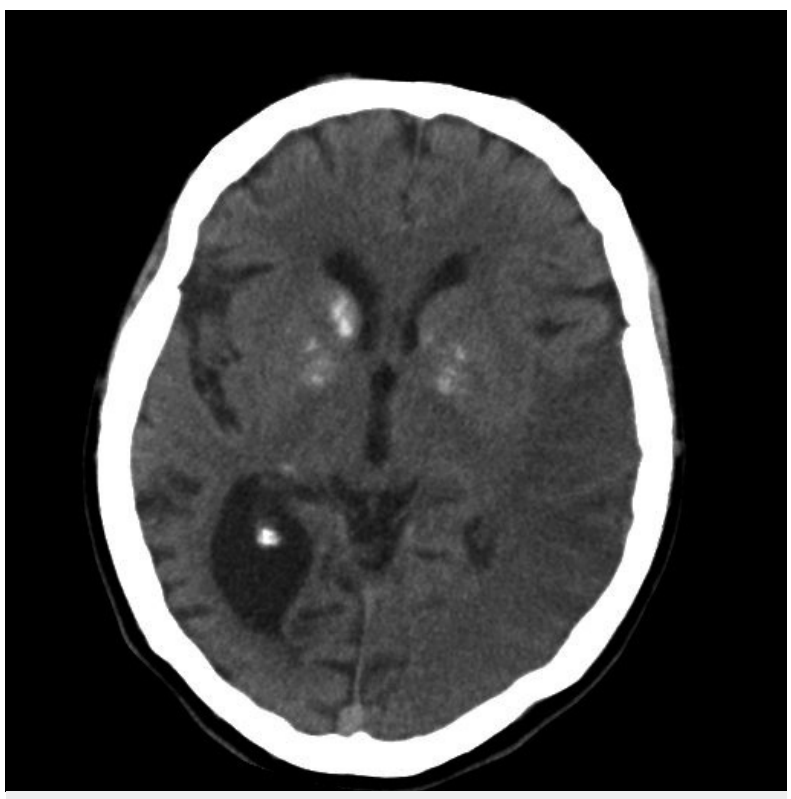

Figura 1. Tomografía de cráneo simple.

gangliobasales evidentes en la escanografía. En conjunto, estos hallazgos plantean como probable etiología una enfermedad mitocondrial, probable MELAS.

Se decide complementar los siguientes estudios:

Suero: ácido pirúvico $0,099 \mathrm{mmol}$ litro $(0,03-0,12$ $\mathrm{mmol} / \mathrm{lt})$, ácido láctico: $1.88 \mathrm{~mm} / \mathrm{lt}(0,5-2.2 \mathrm{mmol} / \mathrm{Lt})$, relación lactato piruvato $18.5(0-25)$. Estudio de ácidos orgánicos: marcado incremento de ácido láctico sin otras alteraciones.

LCR: incoloro, leucocitos 1, proteínas $49.7 \mathrm{mg} / \mathrm{dl}$, glucosa 48,9 mg/dl (suero $85 \mathrm{mg} / \mathrm{dl}$ ), VDRL no reactivo. Ácido láctico en LCR: $5.32 \mathrm{~mm} / \mathrm{lt}(0,2-2.2 \mathrm{mmol} / \mathrm{Lt})$.

A los 7 días de evolución se realizó control de RM cerebro (figura 3) y espectroscopia cerebral (figura 4), encontrando incremento en la extensión de la lesión sin cambio significativo en su comportamiento magnético, con picos prominentes de lactato en la lesión, en el tejido cerebral sano y en el líquido cefalorraquídeo así como disminución de $\mathrm{N}$ - acetil aspartato (NAA) en el área de la lesión.

Con la alta sospecha clínica e imagenológica de síndrome de MELAS, se decidió complementar estudio con biopsia del músculo cuádriceps, reportada dentro de los límites normales. Adicionalmente, se realizó estudio genético positivo para mutación puntual m.3243a $>$ g en el gen mt-tl1 del genoma mitocondrial confirmando el diagnóstico.

A los 4 meses, la paciente consultó por crisis focales clónicas del hemicuerpo derecho por lo que se realiza resonancia magnética de cerebro (figura 5), encontrando nueva lesión cortical hiperintensa en T2 y FLAIR, con restricción de señal en las secuencias de difusión. Se observó disminución significativa del edema en la lesión previa, sin evidencia de una zona de encefalomalacia.

\section{REVISIÓN}

El síndrome de miopatía mitocondrial, encefalopatía, acidosis láctica y episodios que simulan infarto (MELAS, por sus siglas en inglés) es una enfermedad de herencia mitocondrial, en la mayoría de casos causada por mutación puntual en el gen A3243G (1, 2). Clínicamente presenta un espectro dependiente de la heteroplasmía que puede comprender desde un síndrome completo a manifestaciones puntuales, siendo características la encefalopatía y los episodios que simulan infarto (ESI) $(3,4)$.

Las imágenes del SNC acompañadas de la clínica adecuada permiten realizar un diagnóstico de la entidad. En general, la tomografía de cráneo simple es utilizada en el estudio inicial de los pacientes con ESI, con hallazgos compatibles con infarto cerebral dados por hipodensidad cortico subcortical (2). Además, se ha descrito en múltiples pacientes calcificaciones gangliobasales bilaterales $(2,3)$.

La resonancia magnética (RM) de cerebro es el estudio de elección para el diagnóstico de MELAS. Se han descrito dos presentaciones principales, las lesiones que simulan ACV (LSA) y lesiones de la sustancia gris profunda (3). La característica de la LSA consiste en compromiso de predominio cortical, hiperintensa en T2 y FLAIR. En las imágenes de difusión hay restricción y se ha encontrado variación de la señal en el mapa de coeficiente aparente de difusión (ADC), donde hay predominio de lesiones con alta señal compatibles con edema vasogénico y en otros casos, baja señal compatible con edema citotóxico, las cuales pueden estar juntas en un mismo paciente $(4,5)$. La explicación para esta variación es el tiempo de evolución de la lesión y la magnitud de la falla energética. De este modo, el compromiso citotóxico se traduce en una mayor severidad de la enfermedad y su presencia tiene mayor riesgo de secuelas neurológicas $(4,5)$. Respecto a la localización de la LSA, es uni o multifocal, sin respeto por territorio vascular, se ha descrito en $1 / 3$ de los pacientes un inicio focal generalmente en región temporal que en el plazo de 2 a 3 semanas progresa a compromiso parietal y occipital $(1,5)$.

Las lesiones de sustancia gris profunda en RM consisten en hiperintensidades en T1 e hipointensidades en T2, siendo los sitios más comprometidos el globo pálido, núcleo caudado y el putamen (3). Estas lesiones se han descrito en otras enfermedades mitocondriales como el síndrome de Leigh (3). Otros tipos de alteraciones descritas en menor frecuencia en MELAS son las lesiones de sustancia blanca de 


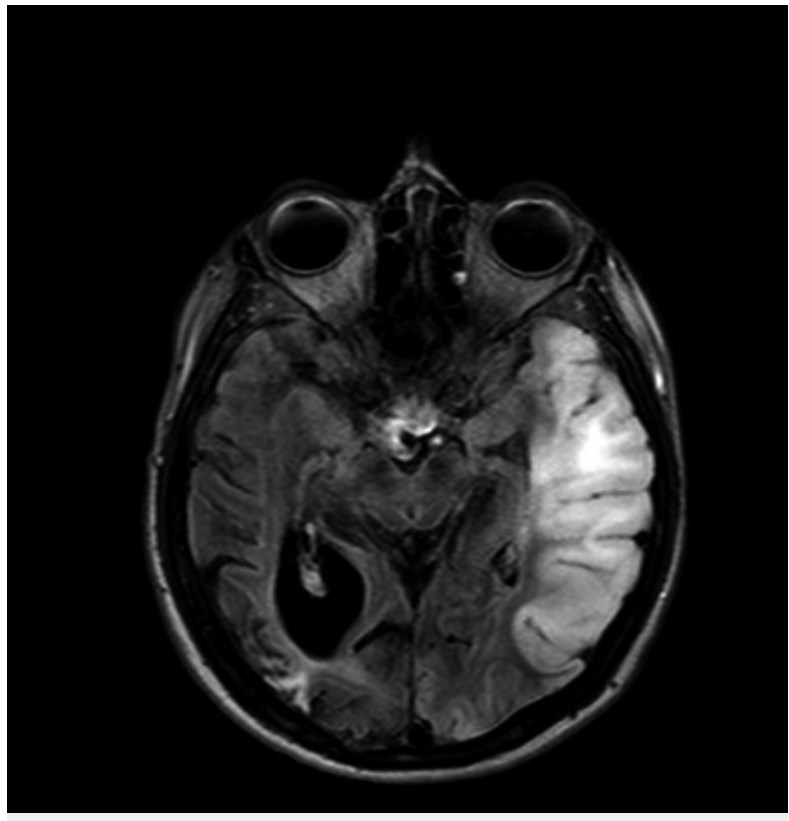

Figura 2. Resonancia magnética de cerebro simple (A) FLAIR.

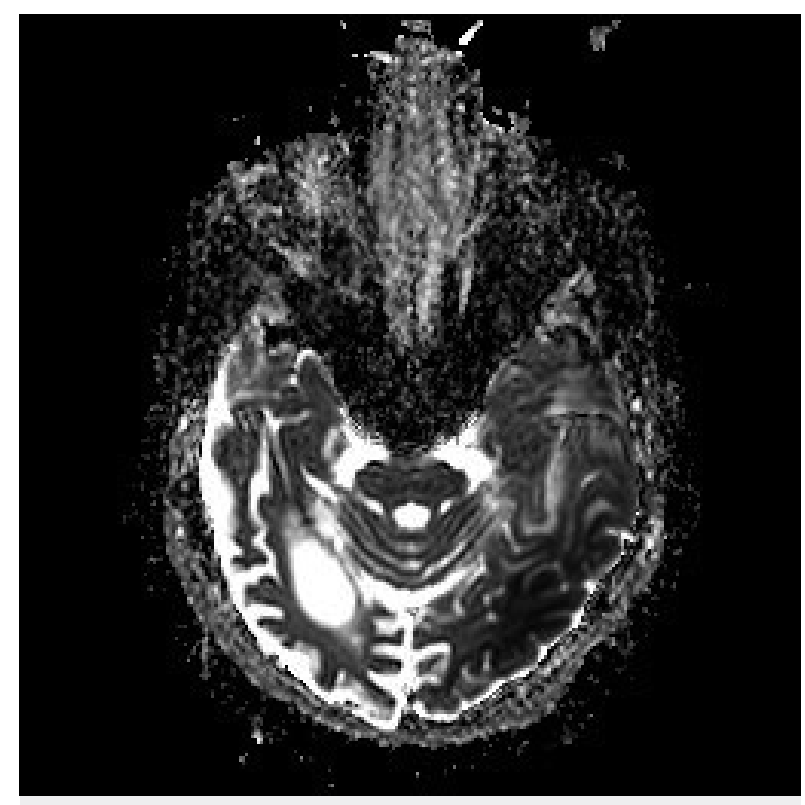

Figura 2. Resonancia magnética de cerebro simple (C) ADC.

predominio en lóbulos occipitales de carácter inespecífico, la atrofia cerebral de predominio en fosa posterior, y menos frecuente, en hemisferios cerebrales (3).

La espectroscopia por RM aporta información complementaria a la RM convencional para el diagnóstico del síndrome de MELAS. En el área del LSA, el comportamiento

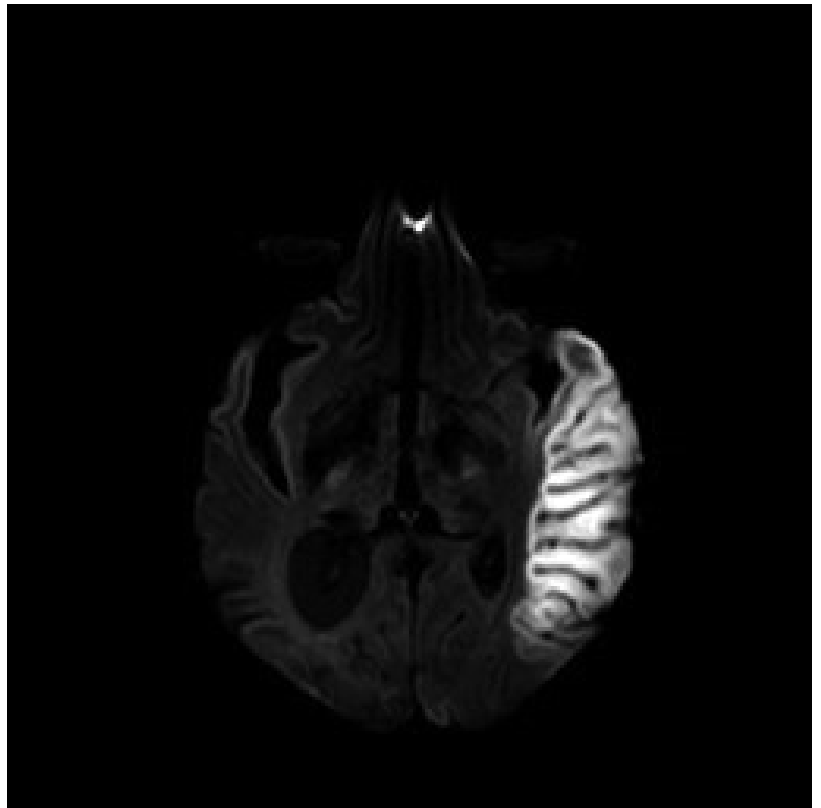

Figura 2. Resonancia magnética de cerebro simple (B) DWI.

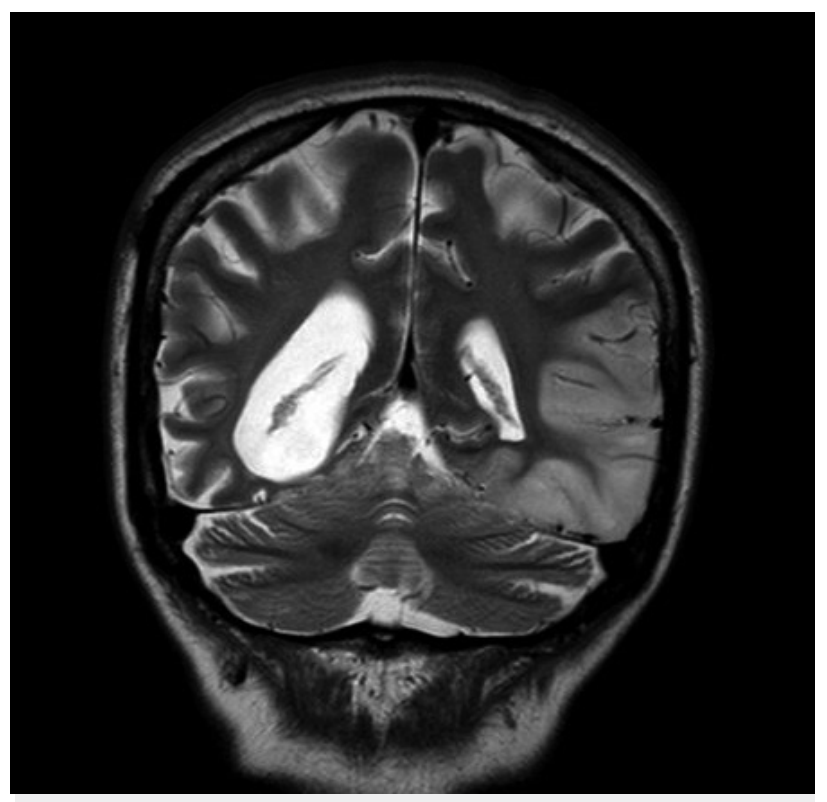

Figura 2. Resonancia magnética de cerebro simple (D) $\mathrm{T} 2$ Coronal.

de los metabolitos es similar al de un infarto cerebral con elevación de lactato y disminución de $\mathrm{N}$-acetil-L-asparato (NAA) (2-5). Sin embargo, en la evaluación espectroscópica del tejido cerebral no lesionado de los pacientes con MELAS se encuentra una elevación de lactato que sugiere metabolismo anaerobio y acidosis global $(2,5,7)$. Se han 


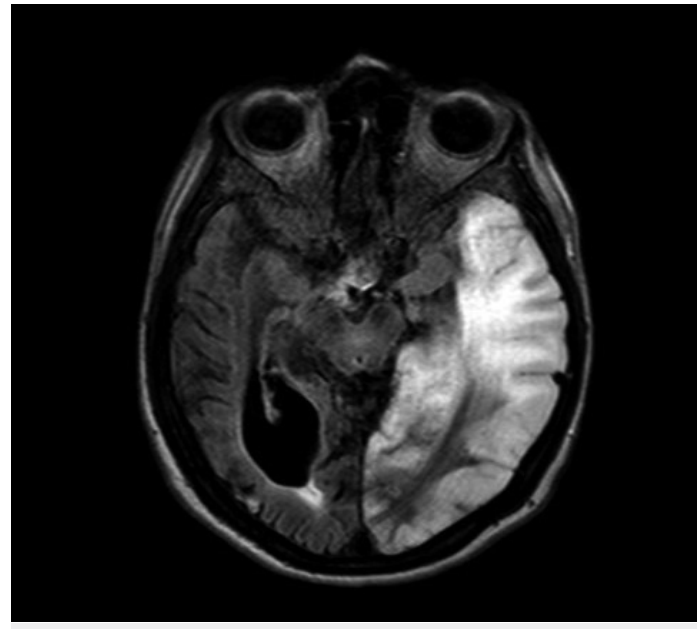

Figura 3. Resonancia magnética de cerebro simple (A) FLAIR.

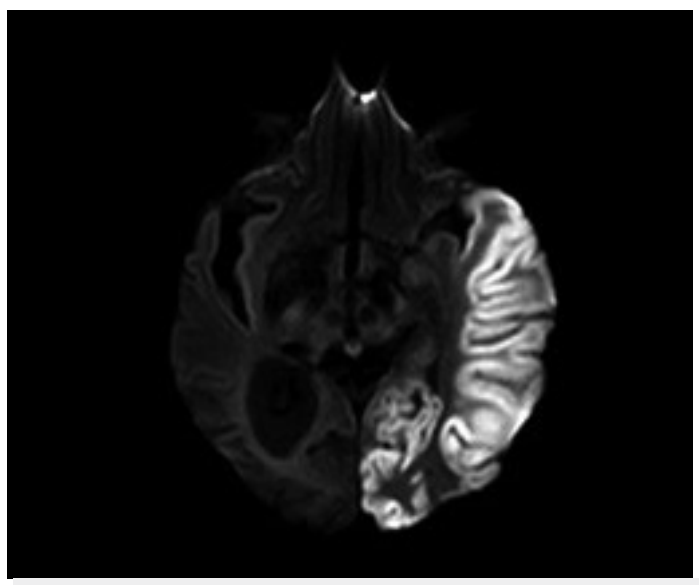

Figura 3. Resonancia magnética de cerebro simple (B) DWI.

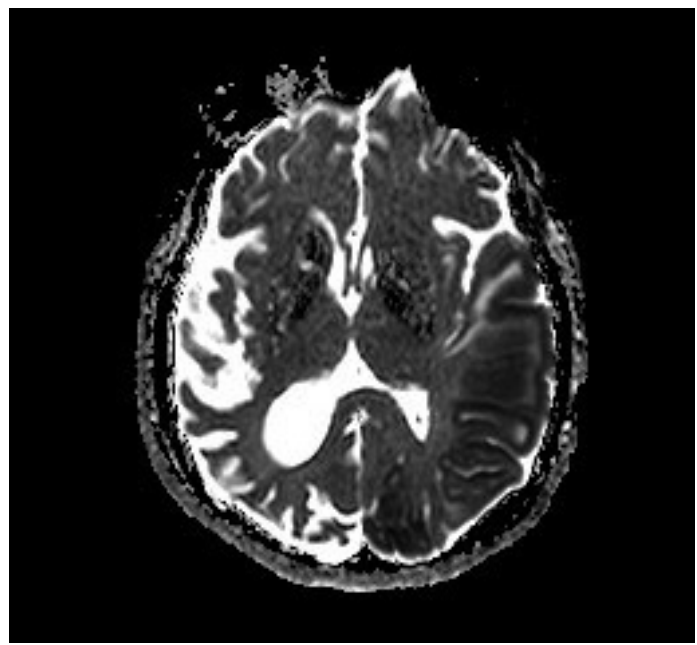

Figura 3. Resonancia magnética de cerebro simple (C) ADC.

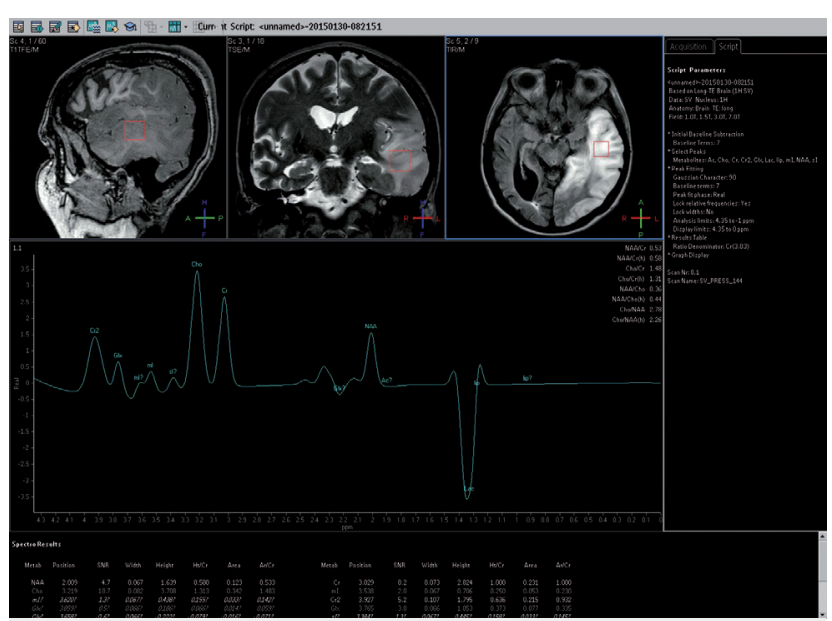

Figura 4. Espectroscopia cerebral por resonancia magnética (A) tejido lesionado.

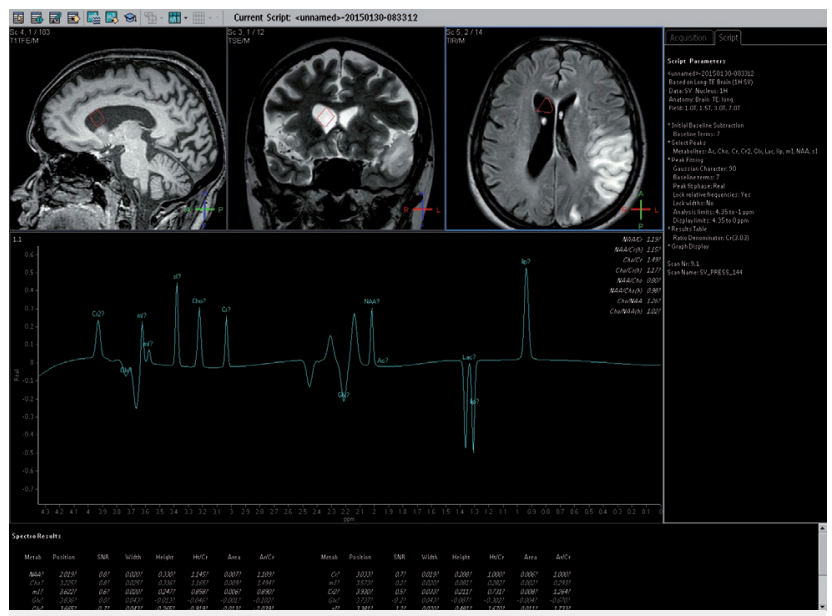

Figura 4. Espectroscopia cerebral por resonancia magnética (B) líquido cefalorraquídeo

correlacionado los niveles de ácido láctico en espectroscopia con severidad de la enfermedad y menor supervivencia (8).

El desencadenante y la patogenia de la LSA son desconocidos. Posibles mecanismos incluyen angiopatía, falla energética o incremento de la demanda de oxígeno por el tejido cerebral. Las imágenes funcionales, además de ser una herramienta diagnóstica complementaria, han permitido describir procesos fisiopatológicos que ocurren durante y posterior a la LSA (3).

Actualmente, las imágenes funcionales por RM por costos y disponibilidad empiezan a reemplazar al PET y el SPECT (7). Estos estudios incluyen las técnicas Arterial Spin Labeling (ASL), la cuantificación de la fracción de extracción de oxígeno (FEO) y medición de la reactividad cerebrovascular (RCV). El ASL es capaz de generar imáge- 


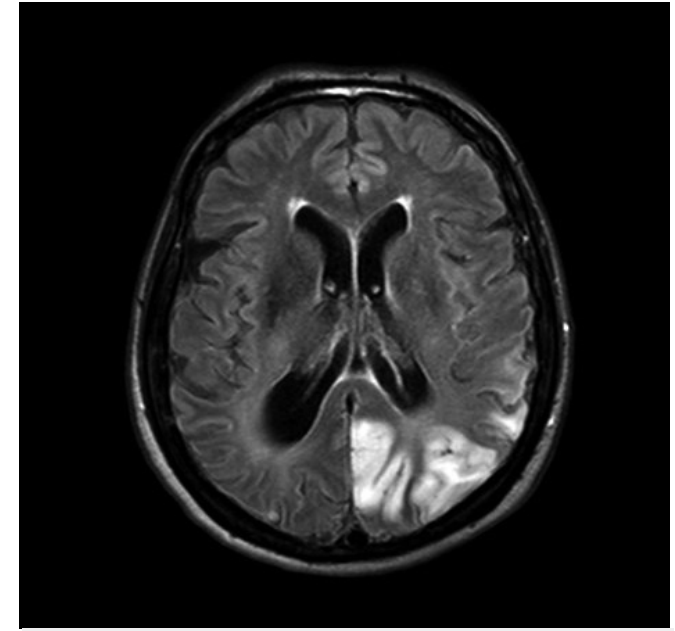

Figura 5. Resonancia magnética de cerebro simple (A) FLAIR.

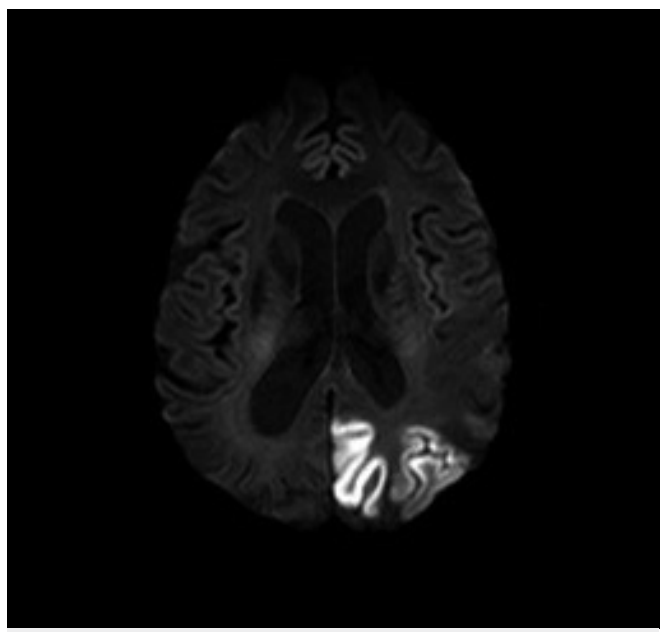

Figura 5. Resonancia magnética de cerebro simple (B) DWI.

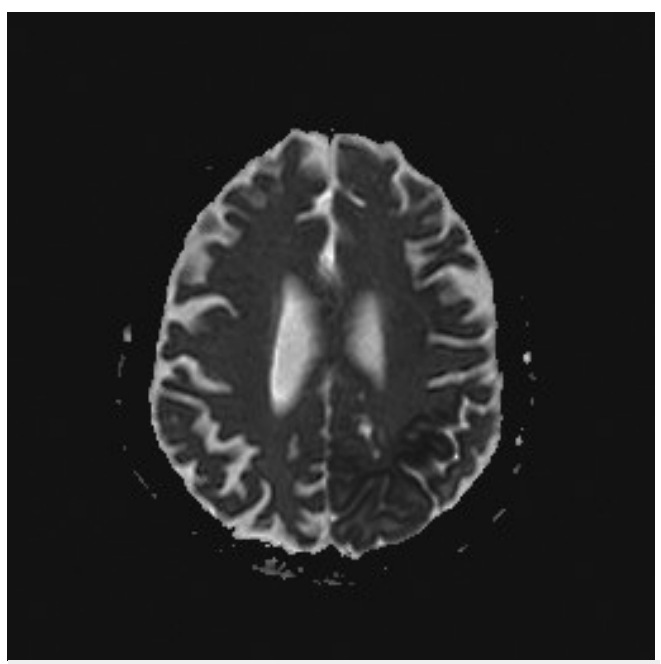

Figura 5. Resonancia magnética de cerebro simple (C) ADC nes de perfusión sin requerir uso de medio de contraste o radiotrazadores $(9,10)$; la hiperintensidad en la imagen indica hiperperfusión y brinda información sobre el flujo sanguíneo cerebral (9). La fracción de extracción de oxígeno está definida como la proporción de oxígeno sanguíneo que un tejido toma del flujo para mantener la integridad funcional y morfológica, reflejando así la eficiencia de utilización del oxígeno (7). La RCV se cuantifica como un cambio en el porcentaje del nivel de oxígeno en la sangre (11).

En los pacientes con MELAS se ha encontrado una hiperperfusión cerebral en la fase aguda y subaguda de predominio en el LSA y una hipoperfusión en el periodo interictal $(12,10)$. Además, está descrito un aumento en la perfusión 3 a 5 meses previo al LSA en el área donde aparece la lesión a pesar de tener una RM (DWI-FLAIR) normal; sugiriendo un valor del ASL como predictor de aparición de lesión (9).

La fracción de extracción de oxígeno, a diferencia del flujo sanguíneo cerebral, está disminuida de forma global en los cerebros de los pacientes con MELAS en todas las fases, asociada a un aumento en la concentración de deoxihemoglobina en el tejido (7). La reducción de la FEO posiblemente se debe a una respuesta adaptativa para compensar el desbalance metabólico como resultado de la inadecuada utilización de oxígeno por el tejido (11). La RCV es inversamente proporcional a los cambios en el flujo sanguíneo cerebral y a la severidad de la presentación clínica (11).

En el presente caso se tienen características típicas clínicas y radiológicas del síndrome de MELAS con episodios que simulan ACV en edad temprana y LSA sin respeto en territorio vascular, predominio cortical, incremento de tamaño en fase subaguda, calcificaciones en ganglios basales y cambios en espectroscopia cerebral compatibles con acidosis global.

\section{Conflicto de intereses}

Los autores manifiestan no tener conflictos de intereses en este estudio. 


\section{REFERENCIAS}

1. Lorenzoni P, Werneck L, Kay C, Silvado C, Scola R. When should MELAS (Mitochondrial myopathy, Encephalopathy, Lactic Acidosis, and Stroke-like episodes) be the diagnosis?. Arq Neuro-Psiquiatr. 2015;73(11):959-967.

2. Le W, Wang Y. Progress in Diagnosing Mitochondrial Myopathy, Encephalopathy, Lactic Acidosis, and Stroke-like Episodes. Chinese Medical Journal. 2015;128(13):1820.

3. Tschampa H, Urbach H, Greschus S, Kunz W, Kornblum C. Neuroimaging characteristics in mitochondrial encephalopathies associated with the m.3243A $>$ G MTTL1 mutation. J Neurol. 2012;260(4):1071-1080.

4. Ito H, Mori K, Kagami S. Neuroimaging of stroke-like episodes in MELAS. Brain Dev. 2011;33(4):283-288.

5. Ramírez S, Marín J, Hernández J, González A, López O, Posso A, et al. Síndrome de Melas: correlación clínica con hallazgos imagenológicos en espectroscopia y tractografía, reporte de caso. Acta Neurol Colomb. 2016(3);32:227-232.

6. Kim J, Lim M, Jeon T, Rha J, Eo H, Yoo S, et al. Diffusion and Perfusion Characteristics of MELAS (Mitochondrial Myopathy, Encephalopathy, Lactic Acidosis, and StrokeLike Episode) in Thirteen Patients. Korean J Radiol. 2011;12(1):15.
7. Xie S. MR OEF Imaging in MELAS. Methods in Enzymology. 2014;:433-444.

8. Weiduschat N, Kaufmann P, Mao X, Engelstad K, Hinton $\mathrm{V}$, DiMauro S, et al. Cerebral metabolic abnormalities in A3243G mitochondrial DNA mutation carriers. Neurology. 2014;82(9):798-805.

9. Ikawa M, Yoneda M, Muramatsu T, Matsunaga A, Tsujikawa T, Yamamoto T, et al. Detection of preclinically latent hyperperfusion due to stroke-like episodes by arterial spinlabeling perfusion MRI in MELAS patients. Mitochondrion. 2013;13(6):676-680.

10. Tsujikawa T, Yamamoto T, Ikawa M, Yoneda M, Kimura H. Crossed cerebellar hyperperfusion after MELAS attack followed up by whole brain continuous arterial spin labeling perfusion imaging. Acta radiol. 2012;53(2):220-222.

11. Rodan L, Poublanc J, Fisher J, Sobczyk O, Wong T, Hlasny E, et al. Cerebral hyperperfusion and decreased cerebrovascular reactivity correlate with neurologic disease severity in MELAS. Mitochondrion. 2015;22:66-74.

12. Wang Z, Xiao J, Xie S, Zhao D, Liu X, Zhang J, et al. MR evaluation of cerebral oxygen metabolism and blood flow in strokelike episodes of MELAS. J Neurol Sci. 2012;323(1-2):173-177. 